\title{
Grundsätze für die Ausarbeitung von grammatischen Minima
}

Zielbewusstes Bemühen um die Ausgestaltung des fremdsprachlichen Unterrichts nach rationellen Gesichtspunkten führt unvermeidlich zur Notwendigkeit einer wohlerwogenen, wissenschaftlich begründeten Auswahl des phonetischen, grammatischen und lexikalischen Lehrstoffes (Minima). Den ersteren zwei Minima ist im Vergleich mit dem Wortschatzminimum bisher verhältnismässig wenig Aufmerksamkeit geschenkt worden ${ }^{1}$. Die folgenden Grundsätze für die Ausarbeitung von grammatischen Minima sind das vorläufige Ergebnis von Arbeiten und Erwägungen einer Arbeitsgruppe der Abteilung für Fremdsprachenunterricht der Tschechoslowakischen Akademie der Wissenschaften ${ }^{2}$.

${ }^{1}$ Von den Arbeiten, die ausschliesslich unsere Frage behandeln, sind die folgenden anzuführen (in zeitlicher Folge): G. M. Voroncova Grammatičeskij minimum dlja srednej školy. In der Zeitschrift: Inostrannyje jazyki v škole, Moskva 1948, No 5.

A. A. Miroljubov $K$ voprosu ob otbore grammatičeskogo materiala dlja srednej školy. Inostrannyje jazyki v škole, Moskva 1956 No 3. Vgl. von demselben Autor Grundprobleme der Fremdsprachenmethodik in der Sowjetschule. Ztschft: Fremdsprachenunterricht, Berlin 1962, No 5, S. 262 ff.

Irmgard Schilling Auswahl und Vermittlung eines grammatischen Minimums im Russischunterricht. Berlin 1956, 142 Seiten.

Antonin Rykl Otázka gramatického minima při vyučováni jazykovým znalostem pro receptivní potrebu. Ztschft: Cizí jazyky ve škole, Praha 1959, No 3.

F. M. Rabinovič $K$ voprosu ob otbore grammatičeskogo materiala. Inostrannyje jazyki v škole, Moskva 1961, No 6.

W. D. Arakin Stoffauswahl und -anordnung bei der Behandlung des grammatischen Systems der russischen Sprache als Fremdsprache. Fremdsprachenunterricht, Berlin 1962, No 7.

Josef Pytelka $K$ otázce výbèru a uspořádání mluvnického minima. Cizi jazyky ve škole, Praha 1962-63, No 6.

G. Gougenheim, P. Michéa, P. Rivenc, A. Sauvageot. L'élaboration du français élémentaire. Etude sur l'établissement d'un vocabulaire et d'une grammaire de base. Paris 1956. S. $206 \mathrm{ff}$.

${ }^{2}$ Katedra jazyků ČSAV, Praha 1 - Nové Město, Opletalova 19, ČSSR. An den erwähnten Arbeiten und Diskussionen nahmen insbesondere folgende Kollegen teil: E. Beneš CSc, L. Dušková, I. Evreinová. 
Wir gehen von der folgenden Definition des grammatischen Minimums (weiter nur GM) aus: GM ist die zweckmässige Auswahl von grammatischen Mitteln einer Sprache, die für die Erreichung eines bestimmten fremdsprachlichen Lernziels notwendig sind.

Das Lernziel wird gewöhnlich (z.B. in Lehrplänen) ziemlich ungenau umrissen. Eine genauere Zielsetzung muss wenigstens folgende Angaben enthalten:

a. Art der $\mathrm{zu}$ erzielenden Sprachbeherrschung (aktiv: nur mündliche Verständigung, oder auch schriftliche Verständigung; passiv: nur Lesen, oder auch Verstehen der gesprochenen Sprache; Übersetzen in die Muttersprache oder Fremdsprache als besondere Fähigkeit);

b. Umfang und inhaltliche Bezogenheit der Sprachbeherrschung (z.B. Verständigung in alltäglichen Situationen, über kulturpolitische Themen usw.);

c. Stufe (Niveau) der Sprachbeherrschung (Lesen mit oder ohne Wörterbuch, geläufiges Sprechen oder nur die Fähigkeit, sich leidlich zu verständigen usw.).

Um möglichst grosse Genauigkeit der Zielsetzung zu erreichen, sollten Angaben dieser Art womöglich auch quantitativ ausgedrückt werden (z.B. Lesetempo, zulässige Fehlerzahl u.ä.).

Aus der Vielfalt aller denkbaren Lernziele ${ }^{3}$ sind für die Unterrichtspraxis wohl die folgenden vier Lernziele (und die ihnen entsprechenden GM) am wichtigsten:

a. Verständigung in den einfachsten und häufigsten Gesprächssituationen. Diesem Lernziel entspräche das elementare GM.

b. Verständigung in allen alläglichen Gesprächssituationen und Lesen leichterer Texte. Diesem Lernziel entspräche das grammatische Grundminimum.

c. Verständigung auch über kulturpolitische und wirtschaftliche Themen, Lesen von mittelschweren belletristischen Texten, Zeitungen und populär-wissenschaftlichen Texten. Diesem Ziel entspräche das „vollständige" GM.

d. Fähikgeit, fremdsprachliche Fachtexte zu lesen. Diesem Ziel entspräche ein spezielles grammatisches Leseminimum.

Bei der Bearbeitung der einzelnen Typen von GM kommt es vorerst prinzipiell nur darauf an, eine solche Auswahl von grammatischen Mitteln zu treffen, die die Verständigung im Sinne des betreffenden Lernzieles ermöglicht. Die Berücksichtigung der pädagogischen und schul-

${ }^{3}$ Vgl. die mannigfache mögliche Zielsetzung bei W. Schtscherba in: Ščerba V. L., Prepodavanije inostrannych jazykov $v$ srednej škole. Obščije voprosy metodiki. Moskva, Leningrad 1947. S. 35 ff. 
organisatorischen Bedingungen muss von der linguistisch-didaktischen Fragestellung und Betrachtungsweise grundsätzlich getrennt werden, da es noch gar nicht erforscht ist, inwieweit die jeweiligen Bedingungen den Lerneffekt beeinflussen.

Die Hauptforderung, die an die Auswahl des GM gestellt wird, ist seine Zweckmässigkeit. Darunter sind folgende Auswahlprinzipien ${ }^{4} \mathrm{zu}$ verstehen:

a. strenge Ausrichtung auf das gesteckte Lernziel,

b. Ökonomie, d.h. Effektivität der Auswahl und Rentabilität der Aneignung.

Ad a. Da die rationelle Auswahl der grammatischen Sprachmittel eben die Erreichung eines bestimmten Lernzieles bezweckt, hat dieses Ziel für die Auswahl eines GM erstrangige Bedeutung, und zwar sowohl in qualitativer, als auch quantitativer Hinsicht. Die Auswahl des GM richtet sich einerseits nach Umfang und Stufe des Lernzieles, d.h. danach, wie weit und wie hoch das Ziel gesteckt ist, andererseits nach der Art des Lernzieles, d.h. danach, ob es sich um ein nur aktives oder nur passives (rezeptives) oder schliesslich um ein kombiniertes aktiv-passives Ziel handelt.

Im Falle eines nur aktiven Zieles mündlicher Sprachbeherrschung, bei der die automatische Verwendung der Sprachmittel im Vordergrund steht, wird sich das GM auf eine ziemlich enge Auswahl von grammatischen Kategorien beschränken können, es wird jedoch eine verhältnismässig grosse Anzahl von formellen Ausdrucksmitteln der betreffenden Kategorien enthalten müssen.

Bei nur rezeptivem Leseziel verschiebt sich das Gewicht eher auf das Verstehen aller systemhaften grammatischen Erscheinungen; wichtiger ist hier vertiefte Kenntnis der Funktionen der Ausdrucksmittel als gedächtnismässige Aneignung aller Einzelheiten der Form. Allerdings treffen diese Forderungen nur dann voll zu, falls unter einem solchen Leseziel das behende Lesen von originalen Texten aller Art mit vollem Verständnis verstanden wird.

Bei kombiniertem Sprach- und Leseziel muss neben einem aktiven Bestandteil (der natürlich gleichzeitig auch rezeptiven Zwecken dient) noch ein zusätzlicher Bestandteil nur für die rezeptive Fähigkeit des Lesens fremdsprachiger Texte angesetzt werden. Zwischen beiden besteht eine enge Verbindung; der rezeptive Bestandteil stellt das Reservoir für

${ }^{4}$ Auswahlprinzipien und Auswahlkriterien unterscheidet bei der Arbeit an dem LM I. D. Salistra Vgl. in dem unter seiner Redaktion entstandenen Slovar' - minimum po nemeckomu jazyku dlja srednej školy, Moskva 1957, S. 7 u. 8; ebenfalls in seiner Methodik des neusprachlichen Unterrichts. Berlin 1962, S. 117 u. 118 . 
die potentielle Bereicherung des aktiven Bestandteils um neue, nun auch aktiv beherrschte Sprachmittel dar.

Ad b) Effektivität des Ziels entbehrliche Mittel ausgeschlossen und umgekehrt alle für die Erreichung des gesteckten Zieles notwendigen Mittel in ausreichendem Masse in das GM aufgenommen werden, so dass mit einem Mindestmass an Mitteln ein Höchstmass an Verständigungseffekt erreicht werden kann.

Die Aneignung der grammatischen Erscheinung muss möglichst rentabel sein. Falls die Wahrscheinlichkeit eines Fehlers oder kommunikativen Versagens infolge der Nichtaufnahme einer grammatischen Erscheinung in das GM minimal ist, ist die Aneignung einer solchen Erscheinung unrentabel. Es ist dabei ausserdem in Erwägung $\mathrm{zu}$ ziehen, dass die Nichtaufnahme einer in der Sprache gering vertretenen Erscheinung zur Herabsetzung der möglichen Fehlerzahl beitragen kann, indem sie die Wahl zwischen zwei funktionell naheliegenden Mitteln ausschliesst.

Durch die Anwendung dieser Prinzipien reiht sich die Arbeit an den Minima in den Bereich der Studien ein, die sich mit der Ökonomie der menschlichen Arbeit befassen. Sie könnte besonders in der Frage nach objektiven Massstäben der Effektivität und Rentabilität, bzw. nach dem optimalen Verhältnis von Effekt und Rentabilität aus Erkenntnissen der Ökonomik möglicherweise methodologischen Nutzen ziehen.

Gegenwärtig wird man bei der Anwendung des Prinzips der Zweckmässigkeit gewisse subjektive Momente bei der Auswahl des GM noch nicht ganz vermeiden können. Man kann und muss jedoch die bisherigen mehr oder weniger intuitiven und willkürlich subjektiven Entscheidungen durch ein rationelles Verfahren ersetzen, nach dem die Auswahl des GM im Sinne verbindlicher, verstandesmässig kontrollierbarer Prinzipien erfolgt und sich dabei auf eine adäquate, wissenschaftlich begründete Sichtung und Wertung des Sprachmaterials stützt.

Bevor wir an die Frage der linguistischen Sichtung und Wertung des grammatischen Materials herantreten, müssen jedoch noch einige Fragen, die aus den Beziehungen zwischen Grammatik und Lexik hervorgehen, geklärt werden. Zwischen beiden besteht, wie bekannt, eine enge Wechselbeziehung. Sie hat unter anderem zufolge, dass die $\mathrm{Zu}$ weisung mancher sprachlichen Mittel, ja ganzer Teilgebiete zur Lexik (Lexikologie) oder Grammatik unklar und strittig bleibt (vgl. die Frage der sogenannten „grammatischen" Wörter, der Komparation, des Aspekts, der Wortbildung u.ä.m.). Da für die didaktische Praxis und auch für unsere Frage der Auswahl des GM letzten Endes nicht wichtig ist, wo diese oder jene Erscheinung registriert wird, ob in der Lexik (im LM) oder in der Grammatik (im GM), kommt es nur darauf an, dass die 
jeweiligen strittigen Erscheinungen nicht von der einen wie auch der anderen Betrachtung und Registrierung übersehen werden.

Wichtiger ist für den Fremdsprachenunterricht die Tatsache, dass manche grammatische Erscheinungen enger an die Lexik gebunden sind, andere dagegen weniger. Diese engere Bindung offenbart sich in der Grammatik in der Form von grammatischen Unregelmässigkeiten und bildet die Grundlage für die Zuweisung solcher Unregelmässigkeiten der sogenannten „lexikalischen" Aneignung (rein gedächtnismässig), der die "grammatische" Aneignung mit Hilfe der Analogie gegenübersteht. Diese Bestimmung der didaktisch zweckmässigen Aneignungsweise hängt ebenfalls nicht von der Lösung der Frage ab, ob wir im linguistischen Sinne mit grammatischen Erscheinungen zu tun haben oder nicht.

Aus der engen Bindung der Lexik und Grammatik geht ferner hervor, dass auch das LM und das GM nicht völlig unabhängig voneinander sein können. Vom linguistischen Standpunkt aus lässt sich schwerlich eine einseitige Abhängigkeit des einen von dem anderen beweisen. Es ist daher wohl angebracht, das LM und das GM anfangs nach eigenen Kriterien aufzubauen; erst sekundär gewinnt die Frage ihrer gegenseitigen Anpassung grosse Bedeutung (z.B. bei der Entscheidung über die Einbeziehung unregelmässiger grammatischer Erscheinungen wird man das Vorhandensein der betreffenden Lexeme $\mathrm{zu}$ beachten haben und umgekehrt wieder müssen die vom Standpunkt des GM wichtigen Erscheinungen auch durch eventuelle nachträgliche Korrekturen im LM in genügendem Masse lexikalisch vertreten werden). Keinesfalls kann jedoch das Prinzip einer etwaigen idealen, d.h. restlos bis in alle Einzelheiten durchgeführten Harmonie des GM und zum entscheidenden Kriterium erhoben werden; dies verbietet der verallgemeinernde, systemhafte Charakter der Grammatik im Unterschied zur Lexik.

Um das im Grunde arbeitsökonomische Prinzip der Zweckmässigkeit auf die Spracherlernung anwenden zu können, muss man die wesentlichen in Betracht kommenden und objektiv feststellbaren linguistischen Eigenschaften des sprachlichen, in unserem Falle grammatischen, Materials beachten und sie mit den schon erwähnten Prinzipien in Beziehung bringen. Die für die Auswahl des GM in Betracht kommenden linguistischen Aspekte, nach denen das grammatische Material in seinen für die sprachliche Kommunikation wichtigen Bezogenheiten gesichtet und gewertet wird, bilden ein engverbundenes Ganzes.

Die ersten zwei Aspekte beziehen sich auf die Sprache als System („langue"); sie beachten 1) die Stellung der grammatischen Hauptfunktionen im System (der funktionelle Aspekt der Systemhaftigkeit), 2) die formellen Ausdrucksmittel der Kategorien vom Gesichtspunkt der Regelmässigkeit der betreffenden morphologischen Formen, wortbildenden 
Modelle und syntaktischen Strukturen (der formelle Aspekt der Systemhaftigkeit).

Der dritte Aspekt beleuchtet das grammatische Material vom stilistischen Standpunkt aus und gleichzeitig von der Asymmetrie von Funktion und Form. Der vierte Aspekt bezieht sich unmittelbar auf die Verwendung der grammatischen Mittel in konkreten kommunikativen Akten (Äusserungen) (,parole), bzw. deren Niederschlag in konkreten Texten) und beurteilt sie nach quantitativen Massstäben (Frequenz). Diese vier linguistischen Aspekte gehen von der jeweiligen Sprache selbst aus. Der fünfte Aspekt hat linguistisch-psychologischen Charakter, denn er betrifft die linguistische Gegenüberstellung des fremdsprachlichen und des muttersprachlichen grammatischen Systems (Übereinstimmung und Nichtübereinstimmung) und die damit verbundenen psychologischen Momente der erleichterten oder erschwerten Aneignung der betreffenden grammatischen Erscheinungen.

Der Aspekt der Systemhaftigkeit, d.h. der Stellung einer grammatischen Erscheinung im System der Fremdsprache, muss zweifellos an die Spitze der linguistischen Gesichtspunkte gestellt werden. Der Terminus „System" wird dabei im Sinne der modernen Sprachwissenschaft als „System der gegenseitig abhängigen und bedingten Sprachmittel" verstanden und nicht in der älteren, jedoch noch immer verbreiteten Auffassung als System aller in der traditionellen Form einer normativen Schulgrammatik angeordneten Sprachmittel.

Der Aspekt der Systemhaftigkeit ist in erster Reihe für die Sichtung der grammatischen Kategorien, vor allem ihrer Funktionen, von Bedeutung, wogegen die formellen Ausdrucksmittel der Kategorien am zweckmässigsten vom Gesichtspunkt der Regelmässigkeit betrachtet werden. Je zentraler und fester eine grammatische Kategorie (Funktion) im System der gegenwärtigen Sprache gelagert ist, desto berechtigter ist ihr Anspruch auf Einbeziehung in das GM.

In welchem Masse systemhafte Erscheinungen in das GM aufgenommen werden sollen, darüber entscheidet das Lernziel. Je weiter und höher das Ziel, desto mehr wird die Auswahl des GM mit dem gesamten grammatischen System zusammenfallen. Bei engerem und niedrigerem Ziel dagegen, also z.B. in dem obenangeführten „elementaren" GM und zum Teil im „Grundminimum", erweist sich eine Auswahl auch bezüglich der systemhaften Kategorien als unerlässlich. Für diese weniger anspruchsvollen Ziele genügt ein Ausschnitt aus dem System, der z.B. nicht alle Tempora, Modi, Genera usw. enthält. Bei der konkreten Auswahl der systemhaften Kategorien werden die im folgenden erörterten Aspekte der Frequenz und der Stilistik herangezogen. Dieselben Gesichtspunkte spielen natürlich auch bei Erscheinungen, die an der Peri- 
pherie des Systems gelagert sind (z.B. absterbende Kategorien, wie der Konjunktiv im Englischen, der Genitiv der Negation im Tschechischen usw.), die nicht in vorhinein bei höheren Lernzielen ausgeschlossen werden können, eine entscheidende Rolle.

Bei der Auswahl der verschiedenen Ausdrucksmittel (morphologischen Formen, wortbildenden und syntaktischen Strukturen), die die systemhaften grammatischen Funktionen bezeichnen, ist uns der Aspekt der Regelmässigkeit behilflich. Die Sprachwissenschaft unterscheidet im Bereich der Form zwischen produktiven und unproduktiven Modellen. Regelmässigkeit kann jedoch auch manchen unproduktiven Modellen nicht abgesprochen werden (nach gegenwärtig unproduktiven Modellen sind manchmal ziemlich viele noch immer gebräuchliche Wörter gebildet oder werden nach ihnen gebeugt). Man könnte solche unproduktive Modelle als ,eingeschränktregelmässig" bezeichnen.

Nach dem Prinzip der Zweckmässigkeit kämen für das GM vor allem die uneingeschränkt regelmässigen (produktiven) als auch die eingeschränkt regelmässigen Formen, die im System der Ausdrucksmittel zentral gelagert sind, in Betracht. Die endgültige Entscheidung muss jedoch bei den letzteren wie auch insbesondere bei den unregelmässigen Ausdrucksmitteln unter Heranziehung des Frequenzaspekts und des stilistischen Aspekt getroffen werden. Die Sichtung des grammatischen Materials von diesem Gesichtspunkt aus ist nicht nur für die Auswahl in das GM, sondern auch für die Aneignungsweise (,lexikalisch" oder „grammatisch") von Bedeutung.

Der stilistische Aspekt ist bei der Auswahl vor allem in den Fällen behilflich, in denen die Sprache mehrere hinsichtlich der Bedeutung mehr oder weniger synonymische Mittel besitzt. Die stilistische Charakteristik: stilistisch merkmallos (neutral) — stilistisch merkmalhaltig (einem bestimmten Stil zugehörig) trägt im Zusammenspiel mit der Häufigkeitscharakteristik und in Abhängigkeit vom Lernziel zur Bestimmung bei, inwieweit neben stilistisch neutralen, allgemein gebräuchlichen Mitteln auch noch stilistisch gefärbte Mittel in das GM aufzunehmen sind. Stilistisch neutral oder merkmalhaltig können auch grammatische Funktionen, insbesondere Nebenfunktionen (in Fällen grammatischer Polysemie), sein, was sich dann zum Teil im Vorhandensein synonymischer Ausdrucksweisen im weitesten Sinne äussert, d.h. in der Möglichkeit, denselben Gedanken formell und stilistisch verschiedenartig auszudrücken (vgl. z.B. das dürfte so sein — das kann so sein — das wird wohl (wahrscheinlich) so sein — das ist wohl (wahrscheinlich) so - u.ä), und die Möglichkeit bietet, auch in solchen Fällen die stilistisch zweckmässigste Ausdrucksweise in das GM aufzunehmen. Bei stilistisch nicht differenzierten Varianten (Doppelformen, aber auch gleichbedeutenden 
Ausdrucksweisen im weitesten Sinne) kann die zweckmässigste Wahl mit Rücksicht auf die grössere Häufigkeit oder (und) Regelmässigkeit der einen oder anderen Ausdrucksweise getroffen werden.

Was die Beziehung verschiedener Typen des GM in stilistischer Hinsicht anbelangt, müssen bei niedriger gestecktem aktivem Lernziel grammatische Synonyme im weitesten Sinne ausgeschlossen werden, bei höheren Typen wird man dagegen auch synonyme Mittel aufnehmen müssen, um die der höheren Zielsetzung entsprechende stilistische Angemessenheit des Ausdrucks zu sichern.

Der Häufigkeitsaspekt ist dazu angetan, Entscheidungen in den Fällen zu ermöglichen, in denen nach den vorhergehenden Gesichtspunkten keine eindeutige Wahl getroffen werden kann, indem er diejenigen grammatischen Erscheinungen aufzeigt, die gegenwärtig besonders häufig in allen Sprachstilen auftreten, bzw. nur für bestimmte Sprachstile typisch sind. Der Häufigkeitsaspekt ist, wie schon angeführt, nicht nur bei Entscheidungen über die Aufnahme von Erscheinungen an der Peripherie des grammatischen Systems, bei unregelmässigen Mitteln und bei der Wahl zwischen grammatichen Synonymen ausschlaggebend, sondern ist auch bei der Auswahl von systemhaften Erscheinungen (bei engem und niedrigem Lernziel) behilflich.

Die enge Verbundenheit der Lexik und Grammatik äussert sich bei diesem Aspekt darin, dass in den meisten Fällen die Entscheidung der lexikalischen Frequenz allein (bei der Auswahl von Vorwörtern, Bindewörtern, unregelmässigen Paradigmen usw.) oder in Verbindung mit der grammatischen Häufigkeitswertung (z.B. bei vereinzelten unregelmässigen Kasusformen) zufällt. Nur bei lexikalisch verhältnismässig uneingeschränkten grammatischen Erscheinungen (systemhaften Kategorien, lexikalisch uneingeschränkten syntaktischen Strukturen) ist nur die grammatische Frequenz allein ausschlaggebend.

Grammatische Häufigkeitszählungen sind bisher nur in geringem Masse vorgenommen worden, und zwar meist nur im Zusammenhang mit der Ermittlung lexikalischer Frequenz. Es muss eigentlich erst die Methodik einer solchen Arbeit geschaffen werden. Vor allem müssten solche Zählungen auch die syntaktischen Strukturen erfassen und die Häufigkeitswerte der einzelnen Bedeutungen (Funktionen) der polysemen grammatischen Mittel ermitteln. Diese Angaben werden für die Aufsteilung der GM um so wertvoller sein, je mehr sie die stilistische Differenzierung der grammatischen Mittel (wenigstens innerhalb der zwei Stilbereiche, der Umgangssprache und der Buch- oder Fachsprache) beachten und festhalten.

Der Aspekt der (Nicht) Übereinstimmung mit der Muttersprache unterscheidet sich grundsätzlich von den bisher angeführten Aspekten, 
die nur von der Fremdsprache ausgingen, indem er die Fremdsprache der Muttersprache gegenüberstellt und die psychologischen Momente der Interferenz des muttersprachlichen Sprachgefühls beim Erlernen der Fremdsprache berücksichtigt.

Vom linguistischen Standpunkt stellen wir bei der Konfrontation zweier Sprachen völlige Übereinstimmungen, völlige Nichtübereinstimmungen und teilweise Übereinstimmungen, der grammatischen Formen und ihres Gebrauchs fest. Die Schlussfolgerung, die wir daraus für das Erlernen der fremden Sprache (in unserem Falle für die Frage des GM) ziehen, sind verschieden im Bereiche der Form auf der einen Seite und des Gebrauchs dieser Formen auf der anderen, unterschiedlich sind zum Teil auch die Schlussfolgerungen bei aktiver und (nur) passiver Zielsetzung.

Bei völliger Übereinstimmung wird man in bestimmten Fällen die übereinstimmende Erscheinung dem Lernenden nicht bewusst vermitteln müssen, was jedoch nicht der Nichtaufnahme in das GM gleichzusetzen ist. In letzterem Falle nämlich wird der Lernende die aus dem GM ausgeschlossene Erscheinung nicht beherrschen, wogegen er in ersterem Falle die Erscheinung auf Grund seiner Muttersprache beherrscht. Im Bereiche der Formen kann dieser Fall nur bei rein rezeptiver Zielsetzung eintreten, und zwar nur dann, falls die betreffenden grammatischen Formen in den beiden Sprachen in lautlicher Hinsicht als gleichwertig identifizierbar sind (dies ist im allgemeinen öfter bei den slawischen Sprachen als z. B. bei den germanischen Sprachen der Fall). Bei aktivem Ziel wird jedoch auch in diesen Fällen die Form in das GM prinzipiell einzuschliessen sein, wobei über die eigentliche Auswahl das „Verfahren" auf Grund der schon früher angeführten Aspekte der Sichtung und Wertung des fremdsprachlichen grammatischen Materials entscheidet.

Bewusste Vermittlung erübrigt sich sowohl bei rezeptiver, als auch bei aktiver Zielsetzung bei völliger Übereinstimmung im Bereich des Gebrauches der Formen (ihrer Funktionen, ihres stilistischen Wertes, ihrer Häufigkeit, lexikalischen Gebundenheit usw.), da die übereinstimmende Gebrauchsweise auf die äquivalente fremdsprachliche Form aus Muttersprache übertragen wird, natürlich nach vorhergehender Gleichsetzung (z. B. auf die folgende Weise: „Die Zukunft wird in ... (der betreffenden Fremdsprache) so ausgedrückt: ..., oder: „Ich werde schreiben (lesen usw.) heisst auf ... so: ...).

Die Reduktion des grammatischen Stoffes um solche nicht bewusst vermittelte Übereinstimmungen kann je nach der Ähnlichkeit der grammatischen Systeme der beiden Sprachen grösser oder geringer sein, sie kann sowohl das Funktionieren der allgemeinsten sprachlichen Kate- 
gorien (Wortarten, Satzteile usw.), als auch das der einzelnen Formen betreffen.

Bei völliger Nichtübereinstimmung ganzer grammatischer Kategorien (vgl. das Fehlen der Kategorie des Artikels in den slawischen Sprachen, des Aspekts in den germanischen und romanischen Sprachen u. ä.) muss man unterscheiden, ob die Kategorie in der Fremdsprache oder in der Muttersprache fehlt. Falls sie nur der Fremdsprache eigen ist, wird über ihre Aufnahme oder Nichtaufnahme in das GM das „Verfahren" nach den fremdsprachlichen Aspekten entscheiden. Ist eine bestimmte Kategorie nur Muttersprache eigen, erweist es sich meist als didaktisch zweckmässig, dem Lernenden gewisse diesbezügliche Hinweise zu geben und ihn auf etwaige Reflexe dieser Kategorie in der Fremdsprache aufmerksam zu machen (vgl. z. B. die Reflexe der Funktion des Artikels in der Wortfolge der slawischen Sprachen, des slawischen Aspekts im Gebrauch der Tempora in den germanischen und romanischen Sprachen usw.). $\mathrm{Ob}$ und inwieweit die Aufnahme dieser Hinweise in das GM zweckmässig ist, kann nur nach der Stellung der Kategorie in der Muttersprache entschieden werden, von der die Wahrscheinlichkeit und Gewichtigkeit eines durch Unkenntnis verursachten Fehlers abhängig ist.

Am häufigsten werden bei der Gegenüberstellung zweiter Systeme teilweise Nichtübereinstimmungen festgestellt. Teilweise Nichtübereinstimmungen im Bereich der grammatischen Formen haben zur Auswahl des GM keine direkte Beziehung. Sie betreffen die Darbietung (Hervorhebung des Unterschieds) und die Einübung (intensivere Einübung der unterschiedlichen Form). Im Bereiche der Funktionen finden wir, dass die Abweichung (neben Gleichheit anderer Funktionen) in der Fremdsprache oder in der Muttersprache oder schliesslich in beiden Sprachen vorhanden ist. Dies verlangt bei der Vermittlung der entsprechenden Formen Belehrungen über die in der Muttersprache nicht vorhandene Funktion oder Warnungen vor Übertragung der in der Fremdsprache nicht existierenden muttersprachlichen Funktion mit eventueller Belehrung über nichtgrammatische Ausdrucksmittel oder grammatische Reflexe in der Fremdsprache. Das Auswahlverfahren für das GM wird in analogischer Weise wie bei den früher erwähnten völligen Nichtübereinstimmungen angewandt.

Dasselbe gilt im grossen und ganzen auch für andere teilweise Nichtübereinstimmungen im Gebrauch der Formen (verschiedener stilistischer Wert, unterschiedliche Frequenz, lexikalische Gebundenheit usw.), die wir unter dem Begriff der "Tiefe" der Vermittlung einer grammatischen Erscheinung zusammenfassen (siehe weiter unten).

Es wurde schon auf die dem Prinzip der Zweckmässigkeit anhaftende Subjektivität hingewiesen. Auch die linguistisch wertenden Aspekte, von 
denen oben die Rede war, sind nicht frei von subjektiven Momenten. Eine Quelle der Subjektivität besteht darin, dass die nach den obenangeführten Aspekten durchgeführte Scheidung der grammatischen Erscheinungen manchmal keine eindeutigen Wertklassen, sondern allmählich abgestufte Wertungen ergibt (vom Zentrum des Systems zur Peripherie, von uneingeschränkter Regelmässigkeit bis zu vollständiger Unregelmässigkeit, von grösster bis zu ganz geringer Häufigkeit). Eine objektivere Scheidegrenze kann hier nur auf Grund möglichst objektiv anwendbarer Massstäbe der didaktischen Effektivität und Rentabilität bestimmt werden. Eine weitere Gefahr subjektiver Wertung tritt dann zutage, wenn die nach den einzelnen Aspekten vorgenommenen Wertungen einander widersprechen. Diese Gefahr kann nur dadurch vermieden werden, dass den einzelnen Aspekten eine objektiv richtige Stellung (Wertigkeit) in der Hierarchie der Aspekte zugewiesen wird. Eine befriedigende Lösung dieses komplizierten Problems wird jedoch wohl erst auf Grund konkreter Arbeit an der Auswahl verschiedener GM und ihrer Verifikation (siehe weiter unten) möglich sein.

Eine sinnvolle Auswahl eines GM kann sich nicht auf eine blosse Aufzählung von grammatischen Erscheinungen begrenzen, sondern muss auch den Umfang der Vermittlung der einzelnen grammatischen Erscheinungen bestimmen. Unter Umfang verstehen wir 1) die Breite, 2) die Tiefe des zu vermittelnden grammatischen Stoffes. Die Breite wird durch die Aufzählung der in das GM aufzunehmenden Formen einer Kategorie (z. B. der Typen der starken Zeitwörter in den germanischen Sprachen) und der Funktionen der einzelnen Formen bestimmt. Die Tiefe betrifft die Aufschlüsse über den stilistischen Wert, lexikalische Gebundenheit, Häufigkeit und andere Aspekte des Funktionierens der grammatischen Mittel. Bei der Festlegung der Breite und Tiefe kommt das schon dargelegte Auswahlsverfahren zur Geltung.

Die Richtigkeit der Auswahl eines bestimmtem GM muss in Anbetracht der ihr anhaftenden subjektiven Momente überprüft werden (Verifikation). Die Aufgabe dieser Überprüfung besteht in der Feststellung, ob die Aneignung des ausgewählten grammatischen Materials die Verständigung im Rahmen des festgelegten Lernzieles mit maximaler Effektivität und mit einer durch die Auswahl des GM selbst verursachten minimalen, vom Standpunkt der Rentabilität annehmbaren Anzahl von Fehlern (Versagen) sichert.

Die Methodik einer solchen Überprüfung, die feststellt, inwieweit das GM die grammatisch richtige Bildung und (oder) die grammatisch richtige Deutung von Sätzen ermöglicht, muss erst geschaffen werden. Es kommen z. B. die folgenden Methoden in Betracht:

a. Untersuchung von Fehlern in freien (mündlichen oder schriftlichen) 
Äusserungen der Schüler darauf hin, ob die vom Lernenden verschuldeten Fehler auf Unkenntnis oder fehlerhafte Anwendung der grammatischen Anweisung, oder aber auf Lücken im GM selbst zurückzuführen sind;

b. Untersuchung von Fehlern in Übersetzungen in die Fremdsprache oder Muttersprache mit ähnlicher Fragestellung;

c. Untersuchung von zusammenhängenden fremdsprachlichen Texten darauf hin, ob und inwieweit die in ihnen vorkommenden grammatischen Erscheinungen im GM enthalten sind.

Die unter b. angeführte Überprüfungsmethode erlaubt ausserdem festzustellen, ob und inwieweit grammatische Erscheinungen verständlich sind, die nicht im GM enthalten sind (rezipierbares Komplement zum GM), die unter a., ob und inwieweit auch mit der Fähigkeit der Lernenden, bestimmte in GM nicht enthaltene grammatische Mittel aktiv zu gebrauchen, gerechnet werden kann (produktives Komplement). Beiderlei Fähigkeit kann das Resultat von verschiedenen Faktoren sein (richtige Übertragung der Funktion auf fremdsprachliche Formen aus der Muttersprache, z. B. der Befehlsfunktion des Infinitivs, mehr oder weniger unbewusste Aneignung von grammatischen Erfahrungen durch häufigen Kontakt mit bestimmten grammatischen Erscheinungen im Lernstoff). Bei nahverwandten Sprachen könnten dieses Komplemente von nicht unbeträchtlichem Umfange sein.

Mit der konkreten Einführung des GM in den Lernprozess hängen aufs engste folgende Probleme zusammen: 1.Anordnung und Reihenfolge des grammatischen Stoffes in den GM, 2. Darbietungsweise der Grammatik.

Unter Anordnung verstehen wir die Aufteilung des grammatischen Stoffes in den einzelnen Typen der GM in der Weise, dass die einzelnen Typen relativ abgeschlossene Teilsysteme, gleichzeitig aber auch ausbaufähige Vorstufen für die höheren Typen von GM bilden. Diese Aufteilung betrifft sowohl unterschiedliche grammatische Erscheinungen, als auch die zyklische Erweiterung und Vertiefung der schon teilweise angeeigneten Erscheinungen. Innerhalb der einzelnen Typen der GM muss weiter die zweckmässige Reihenfolge der Erscheinungen erwogen werden. Es besteht gegenwärtig kaum mehr Zweifel darüber, dass diese Reihenfolge nicht dem "System" der Schulgrammatiken (Darbietung ganzer Klassen von grammatischen Erscheinungen „ein für allemal, gründlich und vollständig") gleichzusetzen ist, sondern dem didaktischen Prinzip der allmählichen Erweiterung und Vertiefung der kommunikativen Fähigkeiten unterstellt werden muss. Die Konkretisierung dieses Prinzips hängt jedoch von einer grossen Anzahl mannigfacher linguistischer und sprachpsychologischer Faktoren und ihrer Wechselbeziehun- 
gen $a b$, ohne deren Erforschung und pädagogisch- experimenteller Überprüfung eine objektive Lösung dieses Teilproblems der Spracherlernung unmöglich ist.

Überaus wichtig ist auch die Lösung des Problems der zweckmässigsten Darbietung der Grammatik (Regel, Modell oder ihre Verquikkung), die gerade in letzter Zeit in den Vordergrund des methodischen Interesses gerückt ist. Zum Problem des GM hat diese Frage zweierlei Beziehung: sowohl für die Überprüfung eines bestimmten GM, als auch für jede praktische Anwendung des GM, sei es in Lehrbüchern oder in „minimalen" Sprachlehren, muss das ausgewählte grammatische Material in konkreten Formen dargeboten und angeeignet (eingeübt) werden. Auch dieses Problem muss eine objektiv begründete Lösung finden, bevor man von der rationellen Lösung des Gesamtproblems der Aneignung der Grammatik wird sprechen können. 